\title{
Coupling of palmitate to ovalbumin inhibits the induction of oral tolerance
}

F.M. O liveira,

A.F. Silva-Neto, C.A. Silva and C.M. Gontijo
Departamento de Bioquímica e Imunologia, Instituto de Ciências Biológicas, Universidade Federal de M inas Gerais, Belo Horizonte, MG, Brasil

\section{Correspondence \\ C.M. Gontijo \\ Departamento de Bioquímica e \\ Imunologia, ICB-UFMG \\ Caixa Postal 486 \\ 30161-970 Belo Horizonte, MG \\ Brasil \\ Fax: + 55-31-441-5963 \\ E-mail: cgontijo@mono.icb.ufmg.br \\ Presented at the XIII Annual Meeting of the Federação de Sociedades de Biologia Experimental, Caxambu, MG Brasil, August 26-29, 1998. \\ Research supported by $\mathrm{CNPq}$ (No. 400421/93.3), FAPEMIG (No. 59/93) and PRPq-UFM G (No. 23072049103/9393). \\ F.M. O liveira was supported by the PIBIC scholarship program (CN Pq). A.F. Silva-N eto and \\ C.A. Silva were supported by the PRO BIC scholarship program (FAPEMIG).}

Received April 9, 1998

Accepted August 3, 1998

\begin{abstract}
Oral tolerance is a phenomenon that may occur in animals exposed to protein antigens for the first time by the oral route. They become unable to produce immune responses at the levels normally observed when they are immunized parenterally with antigen in the presence of adjuvants. Lipids have been used as adjuvants for both parenteral and oral immunization. In the present study we coupled ovalbumin with palmitate residues by incubating the protein with the N-hydroxysuccinimide palmitate ester and tested the preparation for its ability to induce oral tolerance. This was performed by giving $20 \mathrm{mg}$ of antigen to mice by the oral route 7 days prior to parenteral immunization in the presence of $\mathrm{Al}(\mathrm{OH})_{3}$. Mice were bled one week after receiving a booster that was given 2 weeks after primary immunization. Specific antibodies were detected by ELISA. Despite the fact that the conjugates are as immunogenic as the unmodified protein when parenterally injected in mice, they failed to induce oral tolerance. This discrepancy could be explained by differences in the intestinal absorption of the two forms of the antigen. In fact, when compared to the non-conjugated ovalbumin, a fast and high absorption of the lipid-conjugated form of ovalbumin was observed by "sandwich" ELISA.
\end{abstract}

Key words - O ral tolerance

- Lipid-protein conjugates

- Antigen absorption

- Gut mucosa

- Ovalbumin
The gut mucosa represents the principal region through which most of the antigens penetrate the body. This is mainly due to its extensive area, as well as to the presence of different substances derived from food and of the intestinal flora. The absorption of such antigens is mainly due to the presence and activity of M cells which are in close association with the Peyer's patches $(1,2)$. In the local lymphoid tissue, antigens may induce the production of secretory $\operatorname{IgA}$, which also regulates their absorption, as well as a phenomenon called oral tolerance (3-5). In this case, animals that have been orally treated with antigen usually do not produce humoral or cellular immune responses when they are later parenterally immunized, even in the presence of adjuvants (6-8). Many factors can interfere with the occurrence of oral tolerance, such as the nature of the antigen as well as the dose, frequency and time interval between doses (9). There are also other hostrelated factors, such as its immunological history, age and genetic background, that interfere with the phenomenon (8-13).

In most of our studies on the induction of oral tolerance $(5,8,10,13)$ we have chosen B6D2F1 mice (an abbreviation for (C57/B16 
$x$ DBA/2) F1 mice) as hosts and ovalbumin (Ova) as antigen. The possibility of coupling hydrophobic palmitate residues to proteins, which interested us when studying antigen processing and presentation by B cells carrying surrogate antigen receptors (14-17), opened new perspectives for the study of the mechanism of oral tolerance induction. Lipids have been used as adjuvants in many protocols for both parenteral and oral immunization.

The aim of the present study was to determine whether the coupling of palmityl residues to ovalbumin would affect oral tolerance induction in $\mathrm{B} 6 \mathrm{D} 2 \mathrm{~F} 1$ mice of both sexes, obtained from our breeding unit at the Federal University of Minas Gerais, and used when approximately 8 to 12 weeks old. The lipid-ovalbumin conjugates (Ova-palm) were prepared according to a technique previously described by our group (17), although some small modifications were introduced. Briefly, $1 \mathrm{~g}$ of the N-hydroxysuccinimide palmitate ester (Sigma Chemical Co., St. Louis, MO) dissolved in $100 \mathrm{ml}$ of absolute ethanol $\left(55^{\circ} \mathrm{C}\right)$ was mixed with $4 \mathrm{~g}$ chicken egg albumin (ovalbumin) grade V (Sigma) dissolved in $800 \mathrm{ml}$ of phosphate-buffered saline (PBS) with $0.1 \% \mathrm{NaHCO}_{3}, \mathrm{pH} 8$, in the presence of $0.6 \%$ deoxycholate (DOC; Sigma). After overnight incubation at room temperature, the mixture was centrifuged and the precipitated conjugate (Ova-palm) was collected and washed in $50 \mathrm{mM}$ $\mathrm{NaHCO}_{3}$. After freezing in liquid nitrogen, the precipitate was lyophilized.

In order to test the tolerogenic properties of the conjugate, we performed the following protocol for the induction of oral tolerance (by gavage) which we had described previously for ovalbumin (5). Briefly, after being lightly anesthetized with ether, mice were intubated with a urethral polyvinyl catheter calibrated to reach the stomach, and given $20 \mathrm{mg}$ of antigen (Ova or Ova-palm) in $0.3 \mathrm{ml}$ of saline $(0.15 \mathrm{M} \mathrm{NaCl})$ intragastrically (ig). Gavages were performed 7 days prior to parenteral immunization, when mice were then injected intraperitoneally $(i p)$ with $0.5 \mathrm{ml}$ saline containing $10 \mu \mathrm{g}$ Ova or Ovapalm mixed with $1 \mathrm{mg} \mathrm{Al}(\mathrm{OH})_{3}$ as adjuvant. Two weeks later, mice were submitted to the same protocol as a booster, but without $\mathrm{Al}(\mathrm{OH})_{3}$. Mice were bled one week after secondary immunization, and after clotting and centrifugation the serum samples were collected and stored at $-20^{\circ} \mathrm{C}$. The presence of anti-Ova antibodies in the samples was assayed by ELISA procedures. Briefly, polystyrene plates (Nunc, Copenhagen, Denmark) were coated overnight at $4^{\circ} \mathrm{C}$ with $2 \mu \mathrm{g}$ Ova diluted in $100 \mu \mathrm{l}$ coating buffer per well, washed with saline containing $0.05 \%(\mathrm{w} / \mathrm{v})$ Tween-20, saturated with $0.25 \%$ (w/v) casein in PBS, washed again and then coated with serial dilution of mouse antiserum starting at 1:40. After $1 \mathrm{~h}$ at $37^{\circ} \mathrm{C}$, plates were washed, incubated for $1 \mathrm{~h}$ at $37^{\circ} \mathrm{C}$ with goat antimouse globulin antiserum conjugated to peroxidase (Southern Biotechnology, Birmingham, AL), washed and developed by the addition of $\mathrm{H}_{2} \mathrm{O}_{2}$ and ortho-phenylene-diamine (OPD; Sigma); the reaction was interrupted at 20 min by the addition of $\mathrm{H}_{2} \mathrm{SO}_{4}$ at $1 / 20$, and read at $492 \mathrm{~nm}$ in EIA-reader (BioRad, Hercules, CA). The absorbance values obtained in the assays were reported as a score (ELISA*), which represents the mean \pm SEM $(N=4-6)$ of the sums of the absorbance values of ELISA tests run with serum dilutions of $1 / 40$ to $1 / 5120$. This way of expressing the results was equivalent to reporting the area under the titration curves or electing absorbance at one particular serum dilution as representative. The significance of the difference between experimental and control groups was assessed by two-tailed Student $t$-tests. Positive and negative control samples were run on every plate to standardize the assays.

As shown in Figure 1, mice produced the same amount of anti-Ova antibodies after being orally treated with saline and immunized with either Ova-palm or unmodified 
Ova. This happened despite the presence of hydrophobic residues in Ova-palm conjugates, which might modify the shape of the protein and induce the display of a different set of epitopes. This view is supported by the fact that the conjugates are not as soluble as ovalbumin. As expected, mice orally pretreated with Ova displayed significantly lower levels of anti-Ova antibodies after being parenterally immunized with either form of the antigen. On the other hand, oral pretreatment with Ova-palm did not induce tolerance. Both forms of the antigen were able to elicit the production of anti-Ova antibodies at the same level as controls.

Since Ova and Ova-palm conjugates exhibited similar immunogenic characteristics but different tolerogenic properties, we designed a protocol to evaluate the kinetics of their intestinal absorption. Mice were treated orally with both forms and, at different times, $100 \mu \mathrm{l}$ of blood samples were collected from the retro-orbital plexus with calibrated micropipettes (H.E. Pedersen, Copenhagen, Denmark). Samples were immediately diluted in $0.4 \mu \mathrm{l}$ saline and, after clotting and centrifugation, the supernatant was collected for detection of the antigen by "sandwich" ELISA. Briefly, rabbit anti-Ova antibodies were purified by affinity chromatography using sepharose-4B columns (Pharmacia, Uppsala, Sweden) coupled with Ova. These antibodies were used to coat polystyrene plates as described above. After blocking with PBS/casein, washing and addition of supernatants, plates were incubated for $1 \mathrm{~h}$. The same rabbit anti-ova antibodies were biotinylated. Briefly, $2 \mathrm{mg}$ of antibodies and $50 \mu \mathrm{g}$ of biotinyl-N-hydroxysuccinimide ester (Sigma) were dissolved and mixed in 1.0 $\mathrm{ml} 0.1 \mathrm{M} \mathrm{NaHCO}_{3}$. After a 4-h incubation at room temperature, the solution was dialyzed against PBS. The biotinylated antibodies were allowed to react with streptavidin coupled with peroxidase (Sigma) for $1 \mathrm{~h}$ and the reaction was completed as described above. A standard solution of ovalbumin was run in

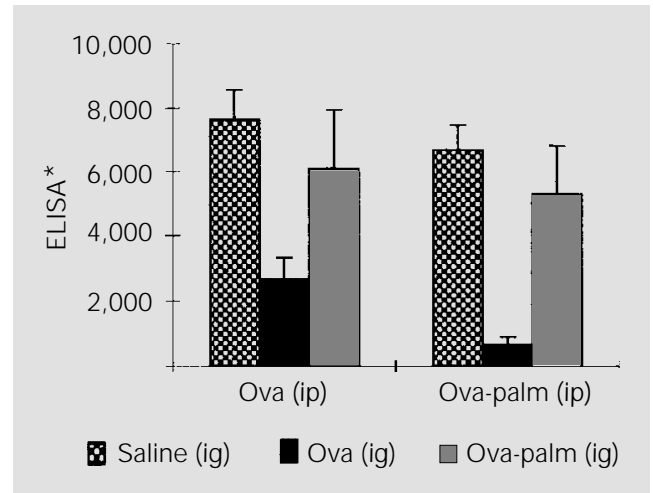

Figure 1 - Failure to induce oral tolerance with Ova-palmitate conjugates. B6D2F1 mice were pretreated with $20 \mathrm{mg}$ of Ova, Ova-palm or saline (in $0.3 \mathrm{ml}$ ), ig, 1 week before being immunized with $10 \mu \mathrm{g}$ of Ova or Ova-palm in $\mathrm{Al}(\mathrm{OH})_{3}$, ip. Two weeks later, mice were submitted to the same protocol as a booster, but without $\mathrm{Al}(\mathrm{OH})_{3}$. Mice were bled one week after secondary immunization. Anti-Ova antibodies were detected by ELISA. Data are reported as a score (ELISA*), which represents the mean \pm SEM $(N=4-6)$ of the sums of absorbance values from serum dilutions of $1 / 40$ to $1 / 5120$.

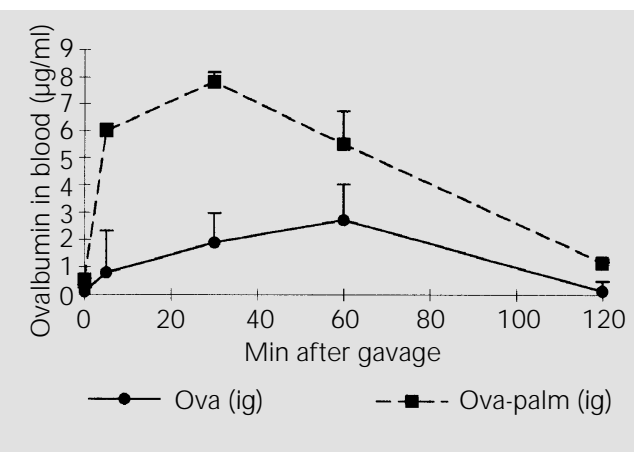

Figure 2 - Kinetics of antigen appearance in blood after intragastric administration. B6D2F1 mice were treated intragastrically with $20 \mathrm{mg}$ of Ova or Ovapalm (in $0.3 \mathrm{ml}$ saline). Animals were bled at several time intervals. Data detected by "sandwich" ELISA represent the mean \pm SEM $(\mathrm{N}=8-9)$ of antigen concentration in blood.

parallel for quantification of the antigen in the samples. As we can see in Figure 2, when compared to mice orally treated with Ova, the antigen was found earlier and at higher levels in the blood of mice that were orally treated with the same amount of Ova-palm.

In the present communication, we have shown that ovalbumin coupled with palmitate residues is unable to induce oral tolerance. On the other hand, its immunogenic property remains the same when administered parenterally. We also noticed that antibodies produced by mice immunized with both forms of the antigen can be equally detected by ELISA using plates coated with either unmodified or palmitate-conjugated ovalbumin (data not shown). These data indicate that the antigenic characteristics, as well as the immunogenic properties, remain the same. 
An explanation for the lack of tolerance induction by Ova-palm conjugates may be related to excess antigen. Antigen doses are crucial for oral tolerance induction (9). Since we observed high levels of intestinal absorption with the conjugates, we tried to induce oral tolerance with the conjugate using lower amounts of the antigen (10-100 times less), but again we were unable to induce oral tolerance (data not shown).

As mentioned earlier in this paper, although little is known concerning their mechanisms of action, lipids have been used as adjuvants for both parenteral and oral immunization. Their use includes the preparation of liposome vesicles, immunostimulating complexes (ISCOMs) and water-in-oil emulsions like Freund's complete and incomplete adjuvants (18-20). We suggest that ovalbumin coupled with lipid residues might induce immune responses instead of tolerance when administered by the oral route.

\section{References}

1. Sneller MC \& Strober W (1986). M cells and host defense. J ournal of Infectious Diseases, 154: 737-741.

2. Neutra MR \& Kraehenbuhl J P (1994). Mucosal immunization via $M$ cells for production of protective secretory IgA antibodies. American J ournal of Tropical Medicine and Hygiene, 50: 10-13.

3. Husband AL \& Gowans JL (1978). The origin and antigen-dependent distribution of IgA-containing cells in the intestine. J ournal of Experimental Medicine, 148: 1146-1160.

4. Challacombe SJ \& Tomasi TB (1980). Systemic tolerance and secretory immunity after oral immunization. J ournal of Experimental Medicine, 152: 1459-1472.

5. Rios MJC, Pereira MAC, Lopes LM, Gontijo CM, Castanheira EB \& Vaz NM (1988). Tolerance and priming initiated by mucosal contact with protein antigen in inbreed strains of mice. Brazilian J ournal of Medical and Biological Research, 21: 825-836.

6. Wells HG (1911). Studies on the chemistry of anaphylaxis. III. Experiments with isolated proteins, especially those of the hen's egg. J ournal of Infectious Diseases, 9: 147-171.

7. Thomas HC \& Parrot MV (1974). The induction of tolerance to a soluble protein antigen by oral administration. Immunology, 27: 631-639.

8. Vaz NM, Maia LCS, Hanson DG \& Lynch J M (1977). Inhibition of homocytotropic antibody responses in adult mice by pre- vious feeding with the specific antigen. J ournal of Allergy and Clinical Immunology, 60: 110-115.

9. Peng HJ , Turner MW \& Strobel S (1989). The kinetics of oral hyposensitization to a protein antigen are determined by immune status and the timing, dose and frequency of antigen administration. Immunology, 67: 425-430.

10. Hanson DG, Vaz NM, Maia LCS \& Lynch J L (1979). Inhibition of specific immune responses by feeding protein antigens. III. Evidence against maintenance of tolerance to ovalbumin by orally-induced antibodies. J ournal of Immunology, 123: 2337-2344.

11. Strobel S \& Ferguson A (1984). Immune response to fed protein antigens in mice. 3. Systemic tolerance or priming is related to age at which antigen is first encountered. Pediatric Research, 18: 588594.

12. Mowat AM, Lamont $A G \&$ Bruce $M G$ (1987). A genetically determined lack of oral tolerance to ovalbumin is due to failure of the immune system to respond to intestinally-derived tolerogen. European J ournal of Immunology, 17: 1673-1676.

13. Vaz NM, Rios MJ C, Lopes LM, Gontijo $\mathrm{CM}$, Castanheira EB, J acquemart $\mathrm{F} \&$ Andrade LAB (1987). Genetics of susceptibility to oral tolerance to ovalbumin. Brazilian J ournal of Medical and Biological Research, 20: 785-790.

14. Gontijo CM \& Möller G (1991). Membrane-incorporated immunoglobulin re- ceptors increase the antigen-presenting ability of B cells. Scandinavian J ournal of Immunology, 34: 577-584.

15. Gontijo CM \& Möller G (1992). Increasing the efficiency of lipid-conjugated antibodies incorporation into the membrane of antigen presenting B cells. Brazilian J ournal of Medical and Biological Research, 25: 909-912.

16. Gontijo CM \& Möller G (1993). Membrane-incorporated surrogate receptors increase antigen presentation by resting $B$ cells, but not by LPS-activated blasts. Scandinavian J ournal of Immunology, 37: 59-63.

17. Gontijo CM \& Möller G (1993). B cells carrying surrogate receptors in their membranes process and present antigen to specific murine T cells. Scandinavian J ournal of Immunology, 38: 565-569.

18. Davis SS, Hunneyball IM, Illum L, Ratcliffe J H, Smith A \& Wilson CG (1985). Recent advances in the use of microspheres for targeted therapy. Drugs under Experimental and Clinical Research, 11: 633-640.

19. Lovgren K \& Morein B (1988). The requirement of lipids for the formation of immunostimulating complexes (iscoms). Biotechnology and Applied Biochemistry, 10: 161-172.

20. Davis SS, Washington C, West P, Illum L, Liversidge G, Stemson L \& Kirsh R (1987). Lipid emulsions as drug delivery systems. Annals of the New York Academy of Sciences, 507: 75-88. 